\title{
Hétérogénéité dans des populations de tomate sous serre et répercussions sur la conduite technique. I. Effets de l'hétérogénéité sur la production de tomate
}

\author{
M Navarrete \\ INRA, systèmes agraires et développement, 16, rue Claude-Bernard, F75231 Paris cedex 05, France
}

(Reçu le 1er août 1994 ; accepté le 3 février 1995)

\begin{abstract}
Résumé - L'objectif de ce travail est de caractériser les effets de l'hétérogénéité d'état végétatif à l'intérieur d'une population de tomate sous serre sur la production, en comparant des populations homogènes et hétérogènes. Les 2 types de plantes obtenus en faisant varier l'âge à la plantation diffèrent par la vitesse de développement, la masse de l'appareil végétatif et le diamètre de la tige, ainsi que par leur mode d'élaboration du rendement. Les différences persistent pendant quasiment toute la culture, ce qui permet de définir un type de plantes "vigoureuses" et un type de plantes «faibles». Dans les populations hétérogènes formées de l'alternance des 2 types, le type "faible» a une croissance végétative réduite par rapport à ce même type en population homogène. Au contraire, le type "vigoureux" a, dans une population hétérogène, une croissance supérieure. Ces résultats mettent en évidence une forte compétition entre plantes. Le rendement par unité de surface est identique dans les populations homogène et hétérogène. En revanche, l'hétérogénéité réduit la qualité de la production, notamment parce qu'elle augmente la dispersion du calibre des fruits.
\end{abstract}

Lycopersicon esculentum Mill = tomate / serre / hétérogénéité entre individus / population / élaboration du rendement

Summary - Heterogeneity in a glasshouse tomato crop and its effect on crop management. I. Effect of heterogeneity on yield. Experiments were set up to characterise the heterogeneity of individual vegetative states within a greenhouse soilless tomato crop, and to study its effects on yield. The heterogeneity of the planting date, for individuals sown at the same date, enabled us to create 2 types of plants. The 'weak' and the 'vigorous' types differ in the development rate, the weight of vegetative part, and the diameter of stem. Thus, their yield is different (number of fruit and mean fruit weight). The differences between the types persist throughout the crop cycle. In a heterogeneous population (ie vigorous and weak types mixed), the vegetative growth of weak plants is much smaller than that of the same plants in a homogeneous population. On the contrary, vigorous plants have a larger growth than in a homogeneous population. These results show a strong interplant competition. Within the range of studied cases, heterogeneity has no effect on yield per area because of the compensating phenomenon between vigorous and weak types. On the contrary, heterogeneity reduces the quality of the production, because the fruit grade distribution is more scattered than in a homogeneous population.

Lycopersicon esculentum Mill = tomato / glasshouse / inter-plant heterogeneity / population / yield formation 


\section{INTRODUCTION}

Dans toutes les populations végétales, cultivées ou non, il existe une hétérogénéité entre individus (stade de développement, état de croissance, production...). Elle a souvent fait l'objet de recherches, pour connaître son origine et/ou ses effets sur la production. Dans les cultures au champ, 2 groupes de facteurs d'hétérogénéité ont été identifiés : l'état initial des individus, graine ou plantule (Abd el Rahman et Bourdu, 1986) et la variabilité spatiale des conditions du milieu (Fleury et Caneill, 1984 ; Boiffin et al, 1992). L'hétérogénéité entre individus joue de façon variable sur la production suivant la nature des organes récoltés. Pour certaines espèces (céréales), le critère important est le rendement, c'est-à-dire la production moyenne de biomasse par unité de surface. Mais, pour d'autres, c'est la distribution de caractéristiques individuelles des organes récoltés : le calibre de nombreux légumes, par exemple la carotte (Benjamin, 1982), le poids individuel des betteraves sucrières, qui détermine leur qualité technologique (Winner et Feyerabend, 1981).

Dans la culture de la tomate (Lycopersicon esculentum Mill) sous serre et en hors-sol, le degré d'hétérogénéité intra-population est fort (des écarts de production entre plantes de $30 \%$ ne sont pas rares). Or la culture demande de nombreuses interventions manuelles (taille des bouquets floraux, des feuilles, des bourgeons, palissage...), qui sont adaptées à la "vigueur" des plantes. Les maraîchers portent un jugement subjectif sur l'état des plantes, grâce à des observations de l'appareil végétatif : diamètre de la tige, couleur et volume du feuillage, hauteur de la plante (Navarrete, 1993) ; ils donnent alors des consignes de travail, qui sont définies en fonction de l'état de "vigueur" moyen de la serre au moment de chaque opération, puis réalisées par le personnel sur une plante après l'autre. Une forte hétérogénéité rend difficile, voire impossible, la définition d'une consigne unique pour toute la serre, mais aussi l'application uniforme de cette consigne par le personnel : il est vraisemblable que, dans ce cas, des opérations choisies en fonction de la "vigueur" moyenne de la serre ne conviennent pas à l'état de chaque plante. On constate d'ailleurs dans certaines serres que des opérations manuelles sont adaptées en fonction de la "vigueur» de chaque plante, d'où le terme de "conduite plante à plante de la population" ; ceci devrait permettre de retrouver une certaine homogénéité dans la serre.
Plusieurs facteurs d'hétérogénéité coexistent dans la culture de la tomate sous serre. Les graines ne sont pas semées directement, mais plantées après une période de levée en pépinière. L'hétérogénéité des individus à la levée peut être réduite par un tri au moment de la plantation, fondé sur une appréciation visuelle de la longueur des feuilles ou de la hauteur de la plante. Cependant, à cause de la faible densité de plantation (environ 2 plants $/ \mathrm{m}^{2}$ ) et de la conduite sur une tige unique (avec élimination systématique des bourgeons axillaires), on peut se demander si une compensation entre plantes peut avoir lieu lorsque des plantes sont cassées ou ont une croissance végétative réduite. L'hétérogénéité initiale évolue ensuite sous l'effet du milieu, mais aussi des interventions manuelles. Grâce aux systèmes de chauffage, d'alimentation hydrominérale, la serre permet de réduire la variabilité du milieu au cours du temps par rapport au plein champ. En revanche, sa structure (charpentes opaques, parois vitrées) accroît la variabilité spatiale de la température et du rayonnement (Baille et Tchamitchan, 1993 ; Graham et al, 1990) ; elle crée notamment de forts effets de bordure. Les nombreuses interventions manuelles sur les plantes occasionnent des stress (plantes cassées, feuilles arrachées, ou seulement secouées) et ne sont pas toujours réalisées de façon identique sur toutes les plantes. Tous ces facteurs ont un effet d'autant plus important sur l'évolution de l'hétérogénéité au cours du temps que les variétés cultivées sous serre sont à croissance indéterminée, et que le cycle cultural est long (jusqu'à 11 mois). De plus, les semis des cultures sous serre ayant lieu à l'automne, la charge en fruits des plantes est maximale en hiver, lorsque le rayonnement est minimal. Ceci entraîne un déséquilibre entre appareils végétatif et reproducteur et une réduction de la vitesse de croissance, qui peut être plus ou moins forte suivant les individus.

Les maraîchers sont donc confrontés chaque jour à l'hétérogénéité des plantes dans la serre et l'existence de la pratique de conduite plante à plante conduit à s'interroger sur les méthodes de mise au point de références techniques pour cette culture. En effet, le test de techniques culturales a toujours lieu sur des parcelles expérimentales le plus homogène possible. Les connaissances produites ainsi pourraient se révéler ensuite difficiles à utiliser par des maraîchers qui, eux, doivent agir sur des parcelles souvent hétérogènes. Une réflexion du même ordre a été conduite sur la vigne (Rives et al, 1966) : la pratique des viticulteurs, qui taillent chaque pied de 
vigne en fonction de sa vigueur propre, conduit les chercheurs à s'interroger sur leur façon de définir un protocole expérimental sur des parcelles qui sont, là aussi, rarement homogènes. Des expérimentations sur la tomate de serre ont été menées pour comprendre les raisons d'être de la pratique de "conduite plante à plante", et notamment savoir si elle permet de réduire l'hétérogénéité de la population et quels sont ses effets sur la production de tomates. Mais pour cela, il faut auparavant avoir étudié les effets de l'hétérogénéité sur la production de fruits (sur le rendement, suivant l'importance des phénomènes de compensation, et sur la qualité, notamment le calibre des fruits). C'est l'objectif des travaux présentés dans cet article. Les expérimentations réalisées reposent sur des populations de tomate où sont maîtrisés l'origine et le degré de I'hétérogénéité, mais aussi la répartition des individus dans l'espace, méthode couramment utilisée pour étudier les effets de l'hétérogénéité (synthèse bibliographique de Trenbath, 1974).

\section{MATÉRIEL ET MÉTHODES}

\section{Dispositifs expérimentaux}

Trois essais ont été conduits en 1989, 1990 et 1991 à la station expérimentale INRA d'Alénya (sud de la France), avec pour objectif de comparer l'élaboration de la production de tomates sur des populations hétérogènes et homogènes. L'hétérogénéité à l'intérieur des parcelles expérimentales est obtenue artificiellement en créant des types de plantes différents (c'està-dire ayant des états de croissance différents à un instant $t$ ), puis en les disposant dans la serre selon un schéma prédéfini. Ces choix garantissent l'existence d'une hétérogénéité reproductible d'une parcelle à l'autre et d'une année à l'autre.

Les graines (variété Capello) sont semées minovembre en pépinière, dans des bouchons de laine de roche, puis repiquées dans des cubes de laine de roche de $1 \mathrm{dm}^{3}$ de volume. Elles sont alors disposées dans la serre, à leur emplacement définitif, sans pour autant être plantées dans le substrat (constitué de pains de laine de roche de $10 \mathrm{dm}^{3}$ de volume).

La culture se fait en doubles rangs (sud-est/nordouest) à une densité de 2,2 plants $/ \mathrm{m}^{2}$. Un double rang est constitué de 2 rangées de plantes, distantes de $50 \mathrm{~cm}$; sur le rang, les plantes sont espacées de 60 $\mathrm{cm}$. Chaque double rang est séparé du suivant par une allée de $1 \mathrm{~m}$ de large.

Pour créer des "types" de plantes qui diffèrent par leur état végétatif, on fait varier la date de plantation réelle dans le substrat, c'est-à-dire le nombre de jours qui séparent la disposition des plants dans la serre de leur plantation dans le substrat. Retarder la plantation est une pratique courante des maraîchers, qui l'utilisent pour "affaiblir» les plantes en début de culture et favoriser une mise à fruit précoce. Cette méthode a été préférée à un tri des plantes à l'intérieur d'une population quelconque en fonction de leur état végétatif, car on était ainsi certain d'obtenir des différences entre plantes qui soient mesurables et reproductibles d'une année à l'autre. Plusieurs travaux confirment que, en maintenant ainsi plus longtemps les plants dans un volume de substrat réduit, la croissance des racines et des feuilles est freinée ; la production est plus précoce, mais le rendement total est plus faible que dans le cas d'une plantation à la date optimale (Cooper et Hurd, 1968). Le type (+) est constitué d'individus plantés à la date optimale (floraison du $1^{\text {er }}$ bouquet), le type $(-)$ d'individus dont la plantation est retardée à la floraison du

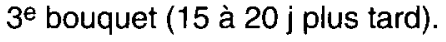

L'essai EXP1 a pour objectif de comparer le comportement des 2 types $(+)$ et $(-)$ en populations "homogènes", que nous appelons respectivement Hom + et Hom - (tableau I). Rigoureusement, ces parcelles devraient être appelées «parcelles d'hétérogénéité minimale» à cause de l'existence d'une hétérogénéité intra-type.

Deux autres essais ont pour objectif d'étudier l'effet de l'hétérogénéité sur le comportement des types (t) et $(-)$. Pour cela on compare des parcelles «homogènes" (Hom) à des parcelles dites "hétérogènes" (Hét), qui sont un mélange en parts égales de plantes des types (+) et $(-)$, disposées en alternance régulière sur le rang (tableau 1) (pour ne pas multiplier le nombre de sigles, les parcelles Hom et Hét portent les mêmes sigles d'un essai à l'autre, bien qu'elles ne soient pas exactement identiques). L'essai EXP2 analyse le comportement du type (+), l'essai EXP3 celui du type (-).

L'essai EXP2 a également pour objectif d'étudier les conséquences de l'hétérogénéité sur la production du couvert. C'est pourquoi nous avons ajouté au dispositif une seconde population hétérogène qui, par construction, est plus proche des populations observées dans les exploitations maraîchères : dans une population d'individus spontanément hétérogène en sortie de pépinière, nous avons réalisé un tri sur la longueur de

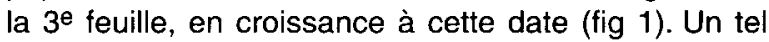
critère a déjà été utilisé pour caractériser l'hétérogénéité d'état végétatif d'une population de betterave sucrière (Fleury et Caneill, 1984). Par ailleurs, sur tomate, la longueur d'une feuille en croissance est un indicateur de la surface foliaire à cette même date (Navarrete, 1993). On postule donc que la surface foliaire du type $(L-)$ est inférieure à celle du type $(L+)$. La population Hét $L$ est formée de l'alternance régulière de 2 types de plantes $\left(L^{+}\right)$et $\left(L_{-}\right)$.

Les plants expérimentaux (blocs et carrés latins) sont construits pour minimiser l'effet de la variabilité spatiale du climat de la serre. Chacun des essais ne regroupe que quelques traitements expérimentaux (tableau I) car les contraintes spatiales sous serre sont fortes. Du fait de la proximité des plantes sur le double rang, donc de l'existence probable d'une compétition à l'intérieur du double rang, on ne peut disposer 2 traite- 


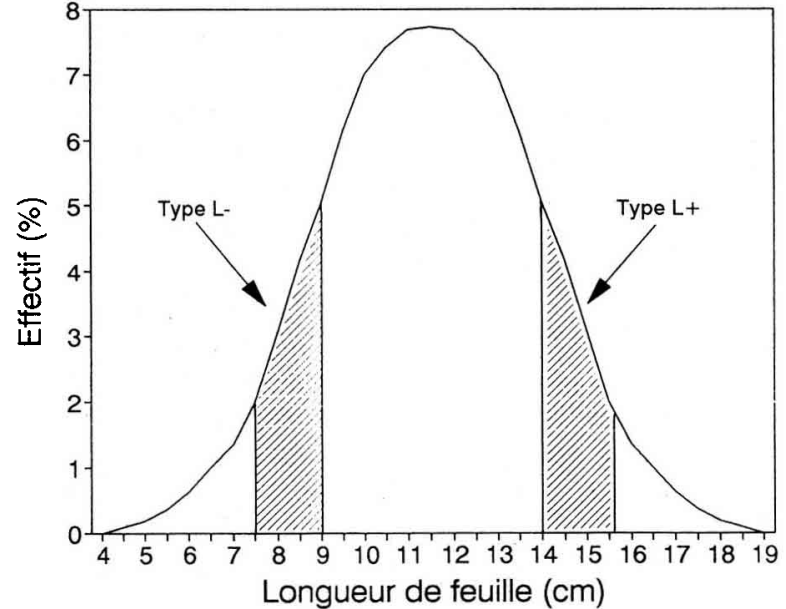

Fig 1. Définition des types de plantes $L+$ et $L-$ au sein de la population de la serre : histogramme de la longueur de la troisième feuille, le 12/12/90 (j28).

ments face à face sur les 2 rangées ; il faut aussi laisser des plantes de bordure de chaque côté de la parcelle expérimentale sur le double rang.

Les techniques manuelles (taille des bouquets floraux, effeuillage, ébourgeonnage) sont réalisées de façon aussi homogène que possible.

\section{Notations et mesures}

Sur les plants de tomate à croissance indéterminée, un nouveau bouquet floral apparaît très régulièrement (toutes les 3 feuilles et tous les 7 à $10 \mathrm{j}$ ). La récolte débute 100 à $120 \mathrm{j}$ après semis, sur le premier bouquet, et s'étale sur 6 à 8 mois, au fur et à mesure de l'arrivée à maturité des bouquets successifs. La plante en fin de culture est constituée d'un empilement de 20 à 30 sympodes (unités formées de 3 feuilles et d'un bouquet). A tout instant, une plante porte environ 8 bouquets, dont les stades de développement s'étagent de la floraison à la récolte.

Sur les parcelles expérimentales, l'état de croissance et de développement est observé tout au long du cycle cultural, par des mesures enregistrées individuellement sur chacune des plantes des parcelles expérimentales. Nous nous sommes limité à des mesures non destructives, sinon le nombre de plantes en fin de culture aurait été réduit très fortement. La date de floraison des bouquets successifs est notée. Nous avons choisi de ne pas mesurer la surface foliaire (qui est forcément destructive compte tenu de la forme et de la dimension des feuilles de tomate), mais d'en faire un suivi indirect : le système de palissage impose un effeuillage progressif en partant du bas de la plante, au fur et à mesure que les bouquets arrivent à maturité. À chaque effeuillage (1 fois/sem), on enregistre la masse sèche des 2 à 4 feuilles éliminées ; leur somme en fin de culture est une estimation de la masse foliai- re produite au cours du cycle. Par ailleurs, sur plusieurs espèces, le diamètre de la tige est corrélé à la biomasse aérienne (sur maïs: Tardieu, 1984), et à la masse sèche foliaire (sur tomate : Navarrete, 1993). La mesure est compliquée sur la tomate à croissance indéterminée : le diamètre du sympode, mesuré à la fin de son allongement, ne diminue pas régulièrement de la base vers le haut de la tige, à cause des changements fréquents de "vigueur» au cours du cycle. C'est pourquoi les mesures de diamètre sont faites sur chaque sympode (environ une dizaine en partant de la base de la plante). La masse surfacique est calculée sur 5 plantes de chaque type $(+)$ et $(-)$ lors d'un effeuillage, comme étant le rapport entre la masse sèche et la surface des limbes des feuilles éliminées à cette date. Les surfaces foliaires sont mesurées à l'aide d'un planimètre vidéo. Les récoltes ont lieu 3 fois par semaine ; à chaque date, on enregistre le nombre et le poids de fruits récoltés sur chaque plante.

D'autre part, on dispose d'un échantillon de 8 plantes (4 de chaque type) situées hors des parcelles expérimentales, mais dans les mêmes conditions climatiques, y compris de compétition due aux autres plantes. Sur cet échantillon, on réalise des mesures destructives afin de caractériser précisément les types $(+)$ et $(-)$ en début de culture. À 2 dates, aux environs de la floraison des bouquets 5 et 9 , sur les essais EXP2 et EXP3, on enregistre le poids sec des organes aériens (feuilles, tiges, fruits), le stade de développement (nombre de feuilles), le diamètre de la tige et la surface foliaire.

Les conditions climatiques (température de l'air dans la serre, rayonnement extérieur) sont enregistrées chaque jour et récapitulées en annexe.

\section{Traitement des données}

Toutes les données étant enregistrées plante par plante, il est possible de calculer l'écart type et la moyenne pour une parcelle donnée, et pour chaque type de plantes.

L'interprétation des essais se déroule en 2 étapes. Dans un premier temps, on étudie le comportement des types (+) et (-) au sein de leurs populations homogènes respectives, puis au sein de la population hétérogène. Ainsi, on caractérise l'effet de l'hétérogénéité intra-population sur l'appareil végétatif et la production de fruits des 2 types de plantes. Ensuite, on étudie le comportement des populations homogènes et hétérogènes. C'est seulement pour cette dernière analyse que les 2 types de populations hétérogènes, Hét et Hét L, sont utilisés. On compare la distribution des biomasses individuelles (moyenne, écart type) de l'ensemble des plantes des populations homogènes et hétérogènes. Ainsi, on caractérise l'effet de l'hétérogénéité intrapopulation sur la production de biomasse du couvert végétal.

Les données sont traitées par comparaison de moyenne et analyse de variance. Comme les plans expérimentaux comportent des traitements non utilisés dans cette analyse, la comparaison des moyennes est 
Tableau I. Tableau récapitulatif des dispositifs expérimentaux.

\begin{tabular}{|c|c|c|c|c|c|c|c|c|c|}
\hline \multirow[t]{2}{*}{ Essai } & \multirow[t]{2}{*}{$\begin{array}{l}\text { Types de } \\
\text { plantes }\end{array}$} & \multicolumn{2}{|c|}{$\begin{array}{l}\text { Traitements } \\
\text { expérimentaux }\end{array}$} & \multirow[t]{2}{*}{$\begin{array}{c}\text { Nb de plantes } \\
\text { par parcelle }\end{array}$} & \multirow[t]{2}{*}{ Dispositif } & \multirow[t]{2}{*}{$\begin{array}{c}\mathrm{Nb} \text { de } \\
\text { répétitions }\end{array}$} & \multirow[t]{2}{*}{$\begin{array}{l}\text { Date de } \\
\text { semis }\end{array}$} & \multicolumn{2}{|c|}{$\begin{array}{l}\text { Date de } \\
\text { plantation }\end{array}$} \\
\hline & & & & & & & & type (+) & type (-) \\
\hline EXP1 & $(+)$ et $(-)$ & $\begin{array}{l}\text { Hom+ } \\
\text { Hom - }\end{array}$ & 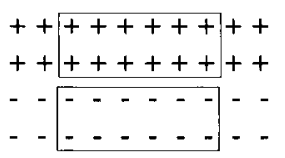 & $\begin{array}{l}12 \text { plantes } \\
\text { sur } 2 \text { rangs }\end{array}$ & Blocs & 3 & $02 / 11 / 88$ & $\begin{array}{c}22 / 12 / 88 \\
(j 50)\end{array}$ & $\begin{array}{c}09 / 01 / 89 \\
(j 68)\end{array}$ \\
\hline EXP2 & $(+),(-)$ & Hom + & $\begin{array}{l}+++++++ \\
+++++++\end{array}$ & & & & & & \\
\hline & $(L+)$ et $(L-)$ & Hét & $\begin{array}{l}+-+-+-+- \\
-+-+-+ \\
-+\end{array}$ & $\begin{array}{l}8 \text { plantes } \\
\text { sur } 2 \text { rangs }\end{array}$ & Carré latin & 3 & $14 / 11 / 90$ & $\begin{array}{c}10 / 01 / 91 \\
(j 57)\end{array}$ & $\begin{array}{c}28 / 01 / 91 \\
(j 75)\end{array}$ \\
\hline & & Hét L & $\begin{array}{l}\cdot 0 \cdot 0 \cdot 0 \cdot 0 \cdot 0 \\
0 \cdot 0 \cdot 0 \cdot 0 \cdot 0 \\
0 \cdot 0\end{array}$ & & & & & & \\
\hline EXP 3 & $(+)$ et $(-)$ & Hom- & $\begin{array}{l}---- \\
+++++\end{array}$ & 3 plantes & Blocs & 5 & 14/11/89 & $15 / 01 / 90$ & $29 / 01 / 90$ \\
\hline & & Hét & $\begin{array}{l}-+-+ \\
+++++\end{array}$ & sur 1 rang & & & & (j62) & (j76) \\
\hline
\end{tabular}

+ : plante du type $(+) ;-$ : plante du type $(-) ; \bullet:$ plante du type $(L+) ; 0$ : plante du type $(L-)$; Hom : populations homogènes, formées des types $(+)$ ou $(-)$; Hét : population hétérogène formée des types $(+)$ et $(-)$; Hét $L$ : population hétérogène formée des types $(L+)$ et $(L-)$; les chiffres entre parenthèses indiquent le nombre de jours après semis. Dans la colonne des traitements expérimentaux, le cadre indique la parcelle; les symboles hors cadres figurent les plantes de bordure.

testée par la méthode des contrastes, plus adaptée que le test de Newmann-Keuls parce qu'elle permet de privilégier les comparaisons de certains traitements tout en conservant la puissance de l'essai complet.

\section{RÉSULTATS}

\section{Comportement des types (+) et (-)}

\section{Comportement des types (+) et ( - ) au sein des populations homogènes (EXP1)}

La vitesse de floraison est constante (fig 2), ce qui s'explique par la relative constance du nombre de feuilles par sympode (caractéristique variétale) et de la température sous la serre (grâce au chauffage et à l'aération). En début de cycle, le type $(-)$ est légèrement en avance par rapport au type $(+)$; la différence est inférieure à $1 \mathrm{j}$ sur les bouquets 1 à 5 , significative à $10 \%$ (tableau Ila). Mais il est ensuite en retard par rapport au type $(+)$; l'écart, d'environ $5 \mathrm{j}$, est significatif et se maintient jusqu'à la fin de la culture.
Le type $(-)$ se caractérise par un diamètre de tige significativement plus faible que le type $(+)$ jusqu'au bouquet 8 (tableau lla). Au-delà, la différence entre types $(+)$ et $(-)$ s'atténue. La masse surfacique du type (-) est légèrement plus faible que celle du type $(+)$ (respectivement 33,2 et $34,5 \mathrm{~g} / \mathrm{m}^{2}$ ) mais l'écart n'est pas significatif.

Ces observations de l'appareil végétatif sont corroborées par les mesures destructives réalisées en début de culture (tableau Ilb). À la floraison du bouquet 9 , on constate que le type $(-)$ a une masse et une surface foliaires plus faibles que celles du type $(+)$. Il en est de même de leurs composantes : nombre de feuilles, masse sèche et surface d'une feuille, ainsi que de la masse et du diamètre de la tige. À la floraison du bouquet 5 (non représenté), les résultats aboutissent aux mêmes conclusions.

L'analyse du rendement au cours du temps permet de tenir compte simultanément de l'évolution du poids des bouquets le long de la plante et de la vitesse avec laquelle les bouquets successifs arrivent à maturité, ce qui dépend principalement de la vitesse de développement. Le nombre de fruits récoltés à une date donnée est supé- 
rieur sur les plantes du type (+) (tableau lla); les différences sont significatives jusqu'à la fin-mai. Le poids moyen des fruits sur l'ensemble de la campagne est légèrement supérieur sur ce type. De ce fait, il est logique que le rendement par plante, qui est le produit du nombre de fruits par leur poids moyen, soit supérieur sur le type $(+)$. L'écart est significatif jusqu'à la fin-mai.

\section{Comportement des types (+) et $(-)$ au sein de la population hétérogène (EXP2 et EXP3)}

Cette analyse se fait par comparaison avec les résultats établis précédemment sur les populations homogènes.
La vitesse de floraison des plantes du type (+) est identique, qu'elles soient en population homogène ou hétérogène. II en est de même pour le type (-).

Pour les plantes du type $(+)$ en population hétérogène (+/Hét, tableau IIla), la croissance végétative n'est pas significativement différente de celle des mêmes plantes au sein de la population homogène $(+/ \mathrm{Hom})$. Cependant, l'écart va toujours dans le même sens : les plantes +/Hét ont un diamètre de tige et une masse foliaire légèrement supérieurs à ceux des plantes $+/ \mathrm{Hom}$. Pour cette dernière variable, le même écart se retrouve pour chaque date d'effeuillage, et il est significatif pour une date (fin avril). C'est pourquoi nous considérons comme

Tableau II. Comportement des types $(+)$ et $(-)$ au sein des populations homogènes (EXP1).

A. Mesures non destructives en cours de culture. Analyse de variance (3 blocs).

Variables

Date de floraison (jours après semis)

Bouquet 3

Bouquet 10

Diamètre de la tige $(\mathrm{mm})$

Moyenne des bouquets 6,7 et 8

Nombre de fruits récoltés par plante (cumul)

Fin mars

Fin avril

Fin mai

Fin juin

Production par plante (kg) (cumul)

Fin mars

Fin avril

Fin mai

Fin juin

Poids moyen d'un fruit fin mai $(\mathrm{g})$
Moyennes

Type (+)

83

143

11,9

12,0

27,3

46,8

61,3

2,1

4,6

7,7

10,2

165

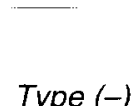

Type (-)

Signification statistique

B. Mesures destructives à la floraison du bouquet 9 (140 j après semis).

Comparaison de moyennes (4 répétitions).

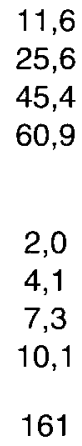

25,6

45,4

60,9

2,0

4,1

7,3

161
NS

$\star \star *$

NS

NS

**

NS

NS
Nombre de feuilles

Masse sèche des fruits $(\mathrm{g})$

Masse sèche des feuilles $(\mathrm{g})$

Masse sèche de la tige $(\mathrm{g})$

Diamètre moyen de la tige $(\mathrm{mm})$

Moyenne des bouquets 2 à 7

Surface foliaire $(\mathrm{m} 2)$

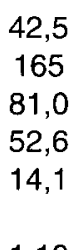

1,10
41

126

62,6

45,0

13,3

1,00
NS

$\star \star \star \star$

**

$\star \star \star$

$\star \star$

* Différence significative à $10 \%$; ** différence significative à $5 \%$; *** différence significative à $1 \%$; NS : différence non significative. 


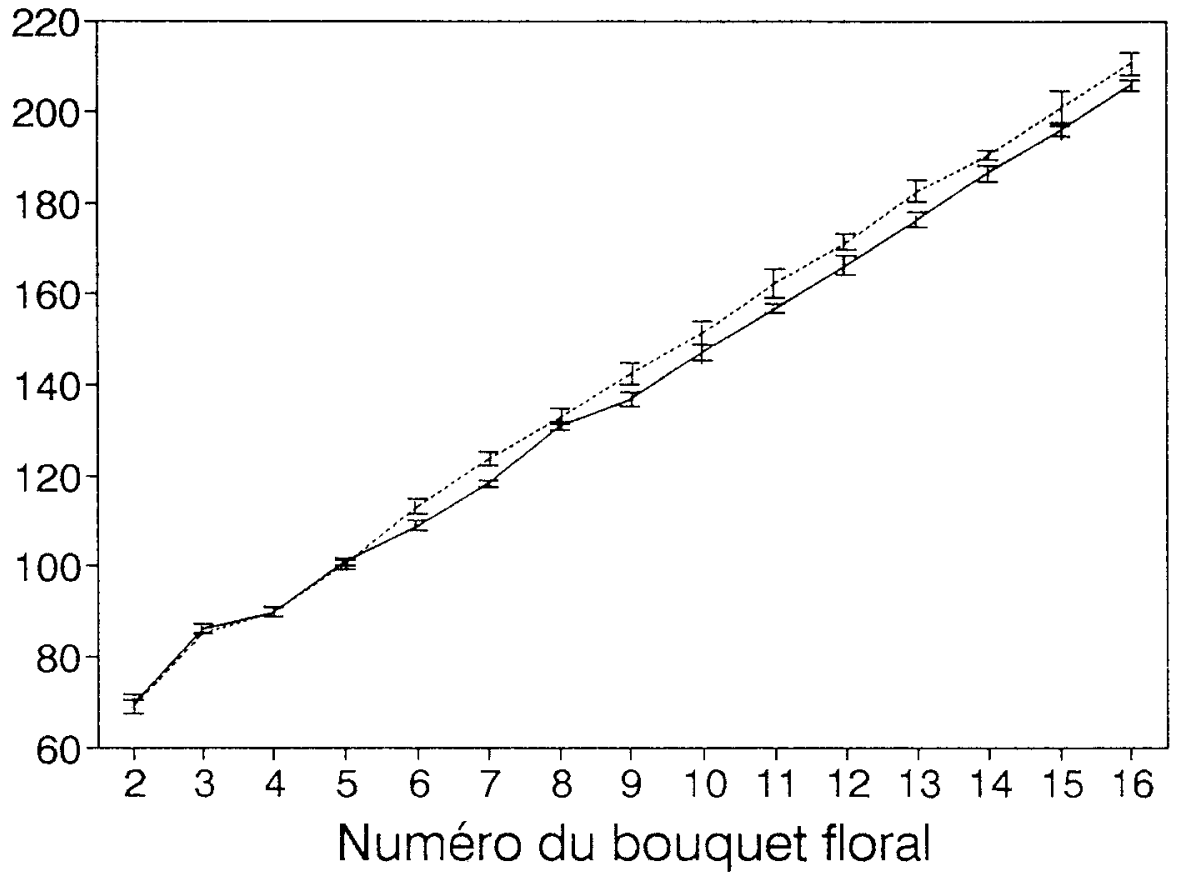

Fig 2. Date de floraison des bouquets successifs pour les types $(+)$ et $(-)$ au sein des populations homogènes (EXP1).

$$
\text { Type (+) ……... Type (-) }
$$

relativement sûr le fait que la masse foliaire des plantes +/Hét soit supérieure à celle des plantes +/Hom. Par ailleurs, les fruits des plantes $+/$ Hét ont un poids moyen plus élevé que ceux des plantes +/Hom (différence significative à $5 \%$ ) ; le nombre total de fruits est en revanche identique sur les 2 traitements. La production totale est légèrement supérieure $(+7 \%)$ sur la population hétérogène, mais de façon non significative. II semble donc que les plantes du type $(+)$ en population hétérogène, c'est-à-dire lorsqu'elles sont entourées de plantes du type (-), aient une croissance végétative et une production de fruits légèrement supérieures à ces mêmes plantes en population homogène.

Pour les plantes du type (-) (tableau IIIb), là encore, bien que peu de différences soient significatives, il semble que l'appareil végétatif et la production des plantes du type (-) en population hétérogène (-/Hét) soit un peu plus faibles qu'en population homogène $(-/ \mathrm{Hom})$. En particulier, le rendement des plantes $(-)$ lorsqu'elles sont entourées de plantes $(+)$ est significativement pénalisé par rapport aux mêmes plantes en population homogène.

\section{Comportement des populations homogènes et hétérogènes}

L'analyse porte successivement sur la dispersion des productions de fruits individuelles à l'intérieur d'une parcelle, puis sur la production moyenne de biomasse du couvert.

Nous comparons les populations Hom+ et Hét grâce aux écarts types intra-parcelle calculés pour le nombre et le poids de fruits par plante (tableau IVa). La population dite «hétérogène», c'est-à-dire par construction, sur des critères d'état végétatif, présente une dispersion des composantes du rendement plus forte que la population dite «homogène». L'écart est significatif à $5 \%$ pour le nombre de fruits récoltés par plante. La distribution du poids moyen des fruits sur la population Hét présente 2 pics (fig 3 ) correspondant aux plantes des types $(+)$ et $(-)$, alors qu'elle est unimodale sur la population homogène $\mathrm{Hom}+$. La distribution sur la population Hét est également plus étalée (présence de fruits de moins de $170 \mathrm{~g}$ et de plus de $210 \mathrm{~g}$ qui n'existent pas dans la population homogène). Cependant, le test de comparaison des 2 distributions (Kolmogorov-Smirnov) n'est pas significatif, probablement à cause du faible nombre d'individus.

En revanche, la production de biomasse végétative du couvert, estimée par la masse moyenne d'une feuille et le diamètre moyen de la tige, n'est pas significativement différente entre la population homogène $(\mathrm{Hom}+)$ et la population hétérogène (Hét) (tableau IVb). II en est de même pour le nombre et le poids de fruits récoltés par unité de surface. Or, les résultats précédents montrent 
Tableau III. Comportement des types (+) et (-) au sein des populations homogène et hétérogène.

A. Type (+) en population homogène (+/Hom) et hétérogène (+/Hét) : essai EXP2

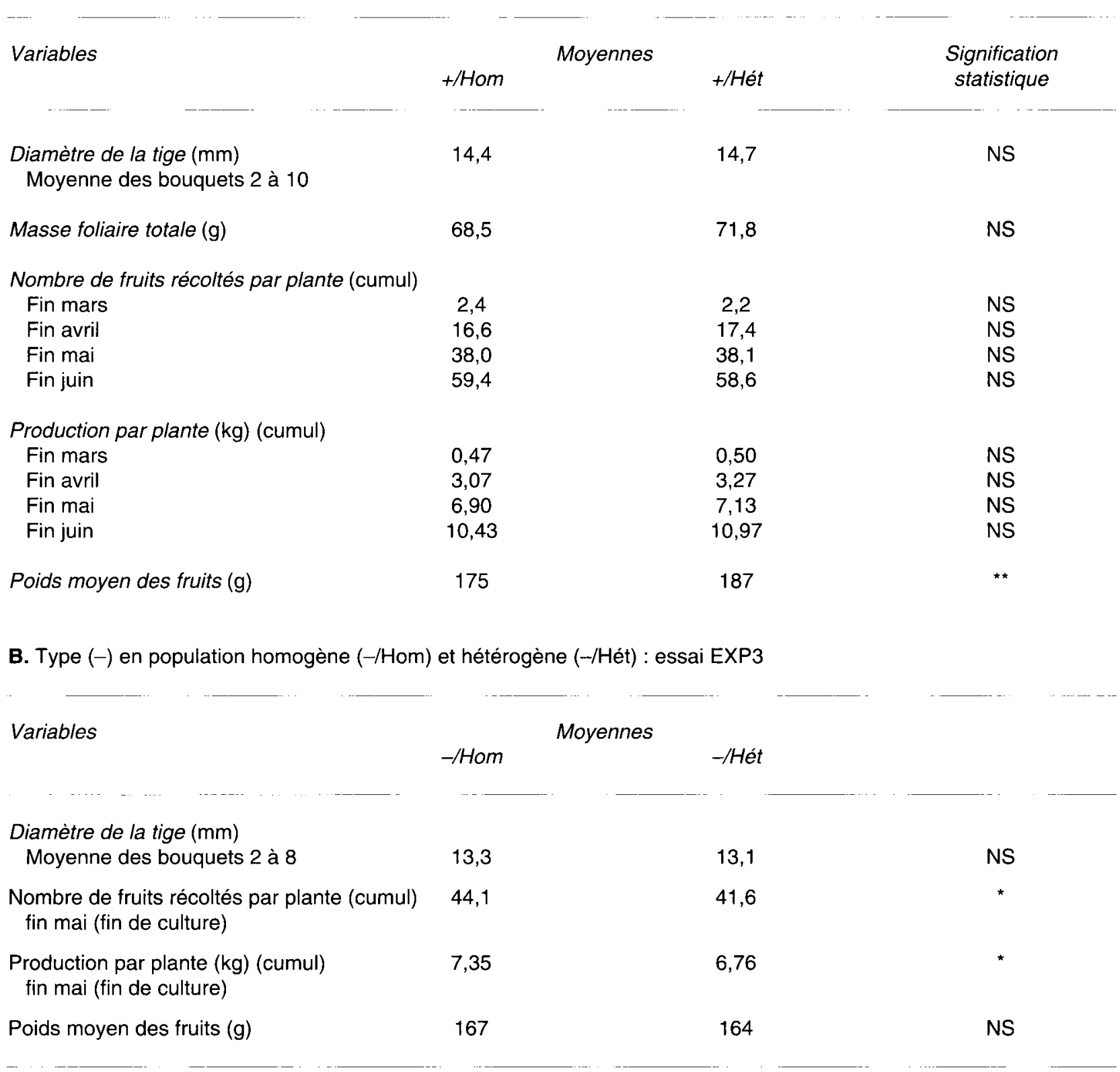

* Contraste significatif à $10 \% ;{ }^{* *}$ contraste significatif à $5 \%$; NS : contraste non significatif.

que le type (+) cultivé en population hétérogène a une production légèrement supérieure à celle qu'il a en population homogène ; au contraire, le type (-) a une production légèrement inférieure à celle qu'il a en population homogène. Dans la population hétérogène, il y a donc eu compensation entre les productions des plantes $(+)$ et $(-)$, au point d'égaler la production de la population Hom+, formée des plantes les plus productives.

Nous ne comparons pas, dans cet article, le comportement des types $\mathrm{L}+$ et $\mathrm{L}-$. Cependant, d'autres résultats (Navarrete, 1993) montrent que leurs états végétatifs respectifs sont bien différents tout au long de la culture, donc que les parcelles Hét $L$ sont bien hétérogènes. Les résultats (tableau IVb) convergent avec ceux obtenus en faisant varier le stade de plantation (parcelles Hét) :

- l'écart type à l'intérieur de la population Hét $L$ est significativement supérieur à celui de la population "homogène», pour la variable «nombre de fruits récoltés par plante» ;

- la production moyenne de la parcelle est identique à celle de la parcelle homogène. 
Tableau IV. Comportement des populations homogène et hétérogène (EXP2).

A. Écarts types intra-parcelle

\begin{tabular}{|c|c|c|c|c|}
\hline Variable & \multicolumn{3}{|c|}{ Écarts types } & $\begin{array}{c}\chi^{2} \\
\text { calculé }\end{array}$ \\
\hline Nombre de fruits récoltés par plante & 4,09 & 5,53 & 6,91 & 6,04 \\
\hline Rendement (kg/plante) & 999 & 1349 & 1290 & 2,22 \\
\hline
\end{tabular}

Test de Bartlett : pour $\alpha=0,05, \chi^{2}$ à 2 degrés de liberté $=5,99$.

B. Moyennes par parcelle

Variable

Diamètre de la tige $(\mathrm{mm})$

Moyenne des bouquets 2 à 8

Masse sèche moyenne d'une feuille $(\mathrm{g})$

Nombre de fruits récoltés par $\mathrm{m}^{2}$

Rendement $\left(\mathrm{kg} / \mathrm{m}^{2}\right)$

\section{$\mathrm{Hom}+$}

14,4

3,17

131

22,9

\section{Moyennes}

Hét

14,2

3,11

128

23,1
Hét $L$

14,1
3,08
123
22,6

Les écarts types et les moyennes pour le nombre de fruits et le rendement sont calculés à partir des données cumulées en fin de culture (fin juin). Résultats de l'analyse de variance : les différences entre populations sont non significatives (probabilité très supérieure à $10 \%$ ).

\section{DISCUSSION ET CONCLUSION}

\section{Discussion des résultats agronomiques}

Le traitement expérimental qui consiste à faire varier la date de plantation permet bien de créer un type $(+)$ formé de plantes «vigoureuses» et un type (-) formé de plantes «faibles»: dans les populations homogènes, le premier a une vitesse de développement et une croissance de l'appareil végétatif (en masse et surface) supérieures. En effet, d'une part, il existe une relation linéaire entre le diamètre de la tige et la masse sèche de l'appareil végétatif (Navarrete, 1993) ; d'autre part, les 2 types de plantes ont des masses surfaciques non significativement différentes. Donc la réduction du diamètre de la tige observée sur le type $(-)$ par rapport au type $(+)$ permet d'affirmer que le type $(-)$ a une masse foliaire plus faible, donc une surface foliaire plus faible; ceci est corroboré par les mesures de surface foliaire réalisées à la floraison des bouquets 5 et 9 . L'effet du traitement initial sur l'appareil végétatif est tout à fait compatible avec la vitesse de développement des plantes, puisque les feuilles ayant une surface réduite sont celles qui étaient en croissance peu après la plantation. La réduction de la croissance végétative entraîne une réduction de la vitesse de développement à partir de la floraison du cinquième bouquet. Tous ces résultats sont en accord avec la bibliographie (Cooper et Hurd, 1968 ; Morgan et Clarke, 1975). Ces derniers interprètent ainsi la réduction de la surface foliaire : en retardant la plantation, la croissance racinaire est limitée au volume du cube, qui, très rapidement, est entièrement colonisé. Les assimilats sont alloués préférentiellement aux autres organes (d'où une vitesse de floraison plus rapide sur les premiers bouquets, que nous avons effectivement observée). La colonisation dans le substrat, hors du cube, a lieu plus tardivement et entre en concurrence avec la croissance des premiers fruits, d'où une diminution de la croissance racinaire, qui, elle-même, provoque une réduction de la surface foliaire. Les différences entre types observées précocement sur la croissance végétative se maintiennent pendant environ 5 mois, 


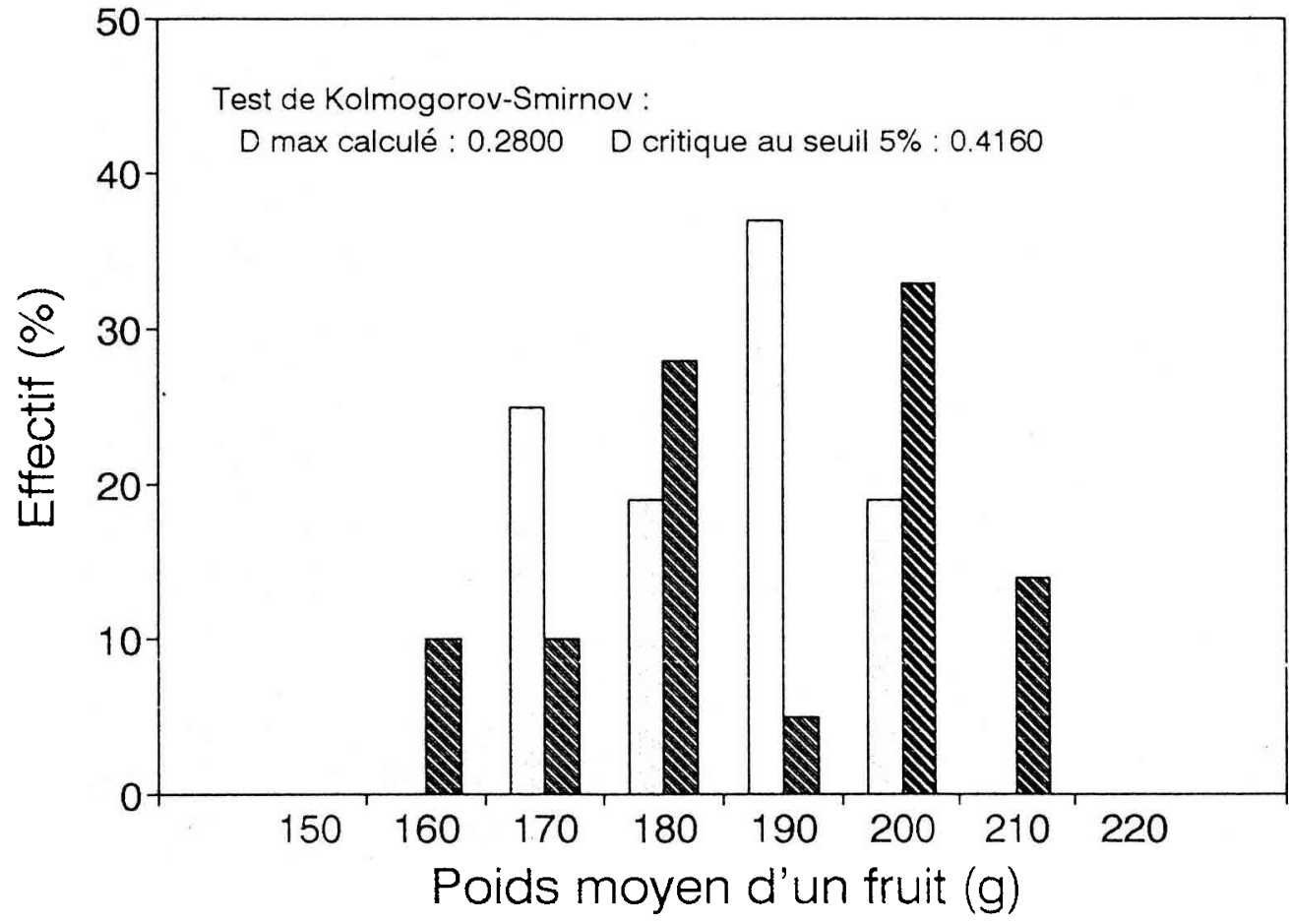

Fig 3. Histogramme du poids moyen des fruits par plante dans les populations homogène et hétérogène (EXP2).

\section{Population Hom + Population Hét}

puisqu'elles se traduisent ensuite par des différences de rendement de même nature.

Le comportement de chaque type dépend également de la nature des plantes voisines, c'est-à-dire de la structure de la population. Ainsi, le suivi de l'appareil végétatif et du rendement au cours du cycle montre une supériorité accrue du type $(+)$ sur le type $(-)$ en population hétérogène, par rapport à ces mêmes types cultivés en population homogène. Des phénomènes similaires de compétition inter-plantes ont été observés par divers auteurs (Glenn et Daynard, 1974 ; Trenbath, 1974 ; Masle et Sebillotte, 1981). Le rendement d'une plante de tomate est donc le résultat de son «potentiel» propre (défini, ici, par le type d'origine) et de son environnement immédiat (ici le type des plantes environnantes). II est probable que c'est la lumière qui est le facteur limitant, bien que cela n'ait pas été vérifié. En effet, la culture a lieu à contre-saison, donc en situation de faible rayonnement. En revanche, les autres facteurs et paramètres du milieu sont jugés satisfisants, car ils sont bien contrôlés par les équipements disponibles dans la serre : culture en hors-sol avec irrigation fertilisante au goutte à goutte, qui garantit un apport régulier et suffisant en eau et en éléments minéraux, enrichissement de l'air en $\mathrm{CO}_{2}$.

La production de matière sèche de la population hétérogène, formée des types $(+)$ et $(-)$ égale celle de la population homogène formée du type $(+)$. Ce résultat semble assez général, puisqu'il est observé sur 2 populations hétérogènes obtenues de façon assez différente (Hét et Hét L). Dans la bibliographie, l'effet de l'hétérogénéité sur la production du couvert est variable, suivant l'importance du phénomène de compensation : dans le modèle de De Wit (Donald, 1963), la compétition entre plantes entraîne une compensation exacte entre les productions des différents individus : l'augmentation de production des plantes dominantes égale la diminution de production des plantes dominées. Chaque individu puise des ressources (notamment la lumière) proportionnellement à son état végétatif (notamment sa surface foliaire). En particulier, si l'indice foliaire est suffisamment important pour capter la totalité de l'énergie incidente, la façon dont cet indice foliaire est réparti entre plantes n'affecte pas le rendement par unité de surface. Cependant, dans d'autres expérimentations, l'hétérogénéité a un effet négatif sur le rendement (Pendleton et Seif, 1962), parce que l'augmentation de production des plantes dominantes est trop faible. Les résultats obtenus sur tomate doivent donc être nuancés : il est possible que, lorsque l'on accroît l'hétérogénéité intra-population au-delà d'un certain seuil (par exemple en augmentant le pourcentage de plantes "faibles", ou l'écart entre plantes "faibles" et "vigoureuses», le rendement diminue, car la totalité de l'énergie lumineuse ne serait plus alors captée. 
Annexe. Conditions climatiques des 3 expérimentations.

\begin{tabular}{|c|c|c|c|c|c|c|c|c|c|}
\hline & Nov & Déc & Jan & Fév & Mars & Avr & Mai & Juin & \\
\hline Rayonnement $^{\text {a }}$ & & & & & & & & & Cumul nov-juin \\
\hline EXP1 & 6,69 & 7,11 & 6,69 & 10,45 & 15,26 & 15,88 & 20,90 & 22,15 & 3183 \\
\hline EXP2 & 7,32 & 6,27 & 6,48 & 8,36 & 11,70 & 19,86 & 20,06 & 21,53 & 3075 \\
\hline EXP3 & 5,43 & 4,18 & 6,90 & 8,78 & 15,68 & 16,72 & 21,32 & 19,23 & 2977 \\
\hline Température $\mathrm{b}$ & & & & & & & & & Moyenne nov-juin \\
\hline EXP3 & 18,1 & 17,0 & 17,3 & 16,9 & 17,7 & 17,5 & 18,9 & 20,3 & 17,9 \\
\hline
\end{tabular}

\footnotetext{
a Rayonnement global extérieur, en MJ.m $\mathrm{m}^{-2} . \mathrm{j}^{-1}$ (en MJ.m $\mathrm{m}^{-2}$ pour la dernière colonne) ; b température moyenne de l'air à l'intérieur de la serre, en ${ }^{\circ} \mathrm{C}$ (du fait de la faible variabilité inter-année de la température sous serre, cette variable n'est indiquée que pour une année d'expérimentation).
}

En revanche, si l'hétérogénéité des populations de tomate ne semble pas entraîner systématiquement une perte de rendement, elle accroît l'hétérogénéité de la production de fruits, et notamment du poids moyen des fruits, car les plantes "vigoureuses" produisent des fruits plus gros que les plantes "faibles".

\section{Conséquences pour les maraîchers de l'hétérogénéité à l'intérieur de leurs serres}

L'augmentation de la dispersion du poids moyen des fruits et donc de leur calibre a des conséquences importantes pour les maraîchers, car elle réduit la qualité globale de la production. En effet, les fruits de petite calibre $<57$ $\mathrm{mm}$ ) sont vendus à un moindre prix, et sont même non commercialisables en dessous d'un certain seuil $(47 \mathrm{~mm})$. D'autre part, la dispersion des calibres accroît le temps de calibrage et de conditionnement des fruits, critère important pour les maraîchers car ces opérations se font sur l'exploitation même. Le poids moyen des fruits a été le seul critère de qualité enregistré. Mais il est possible que d'autres critères de qualité soient également liés au degré d'hétérogénéité de la population (durée de conservation des fruits, fruits déformés...). Or, dans un contexte actuel de saturation du marché de la tomate, la qualité est amenée à jouer un rôle de plus en plus important.

Ces résultats expliquent pourquoi les maraîchers effectuent, en début de culture, un tri sur la "vigueur" des plantes. Cependant, l'intérêt de ce tri doit être relativisé, puisqu'une hétérogénéité supplémentaire se développe au cours du cycle. En revanche, on comprend mieux pour- quoi certains maraîchers adaptent certaines techniques manuelles à l'état de chaque plante ; ils cherchent ainsi à réduire l'hétérogénéité d'état végétatif et donc par conséquence la variabilité du calibre des fruits. L'étude expérimentale de ces pratiques fera l'objet d'un second article.

\section{REMERCIEMENTS}

L'auteur remercie B Jeannequin et M Sebillotte pour leur aide dans la conception des protocoles et l'interprétation des résultats, ainsi que le personnel de la station INRA d'Alénya pour le suivi des essais.

\section{RÉFÉRENCES}

Abd el Rahman N, Bourdu R (1986) Effet de la taille et de la forme des grains sur quelques caractéristiques du développement du maïs au stade jeune. agronomie 6, 181-186

Baille A, Tchamitchian M (1993) Solar radiation in greenhouses. In : Crop Structure and Light Microclimate. Characterization and Applications (C Varlet-Grancher, R Bonhomme, H Sinoquet, eds), INRA, Paris, 93-105

Benjamin LR (1982) Some effects of differing times of seedling emergence, population density and seed size variation in carrot populations. J Agric Sci Cam 98, 537-545

Boiffin J, Durr C, Fleury A, Marin-Laflèche A, Maillet I (1992) Analysis of the variability of sugar beet (Beta vulgaris L) growth during the early stages. I. Influence of various conditions on crop establishment. agronomie 12, 515-525

Cooper AJ, Hurd RG (1968) Effects of planting stage on fruiting of glasshouse tomatoes. J Hortic Sci 43, 167-173 
Donald CM (1963) Competition among crop and pasture plants. Adv Agron 15, 1-118

Fleury A, Caneill J (1984) Croissance et développement de la betterave sucrière. Analyse de l'hétérogénéité du peuplement. In : Écophysiologie de la plante entière, INRA, Paris, 201-212

Glenn FB, Daynard TB (1974) Effect of genotype, planting pattern, and plant density on plant-to-plant variability and grain yield of corn. Can J Plant Sci 54, 323-330

Graham MED, Dubé PA, Phenix M (1990) Measurement of natural irradiance in greenhouses: the effect of averaging period and number of sensors on measurement reliability. Biotronics 19, 83-91

Masle J, Sebillotte M (1981) Étude de l'hétérogénéité d'un peuplement de blé d'hier. I. Notion de structure du peuplement. agronomie 1, 207-216

Morgan JV, Clarke EL (1975) Influence of stage of development at planting on flowering and fruiting in tomato. Acta Hortic 51, 131-145
Navarrete M (1993) Variabilité au sein d'un peuplement de tomate sous serre et répercussions sur la conduite technique. Analyse expérimentale et étude des pratiques culturales des maraîchers. Thèse, université d'Orsay, $152 \mathrm{p}+$ annexes

Pendleton JW, Seif RD (1962) Role of height in corn competition. Crop Sci 2, 154-156

Rives M, Casteran P, Leclair P (1966) Observations préliminaires sur la détermination objective de la taille chez la vigne. CR Acad Agric Fr 52, 537-544

Tardieu F (1984) Étude au champ de l'enracinement du maïs. Influence de l'état structural sur la répartition des racines, conséquences sur l'alimentation hydrique. Thèse, INA-PG, Paris, $232 p+$ annexes

Trenbath BR (1974) Biomass productivity of mixtures. Adv Agron 26, 177-210

Winner C, Feyerabend I (1971) Ein Beitrag zur Morphologie und technischen Qualität des Rübenkopfes. II. Verteilung qualitätsbestimmender Stoffe in der Rübe. Zucker 24, 35-43 